\title{
ANATOMICAL VARIABILITY OF CUTANEOUS NERVES OF ANTERIOR FEMORAL REGION IN HUMAN FETUSES
}

DOI:10.36740/WLek202102106

\author{
Pavlina V. Hryhorieva, Tatiana V. Khmara, Alina 0. Palamar, Tetyana B. Sykyrytska, Maryna Yu. Leka \\ HIGHER STATE EDUCATIONAL INSTITUTION OF UKRAINE "BUKOVYNIAN STATE MEDICAL UNIVERSITY», CHERNIVTSI, UKRAINE
}

\begin{abstract}
The aim: Is to find out the features of innervation of the skin of the anterior femoral region and the fascia lata during the fetal period of human development.

Materials and methods: The study was carried out on 64 preparations of the lower extremities of human fetuses of 4-10 months using macromicroscopic preparation and morphometry. Macropreparations of the skin nerves of the lower extremities of different age fetuses with anatomical variants were subject to photo documentation.

Results: The features of cutaneous nerve fetal topography of the anterior femoral region and the broad fascia of the femur were revealed, their connections were established, and their layering was determined. It was found that in human fetuses, not only the lateral cutaneous femoral nerve but in most cases the branches of other nerves of the lumbar plexus, except for the obturator nerve, are directed to the skin of the anterior-lateral femur surface. The innervation of the medial femur surface is provided by the following nerve complex: obturator, femoral, saphenous and genitofemoral nerves.

Conclusions: Taking into account the fact that the terminal branches of adjacent cutaneous nerves of the femoral region intersect and overlap, innervation bypasses are formed, due to which, in case of possible damage to one of the nerves, its insufficiency is compensated to a certain extent.

Anastomoses were found between the cutaneous nerves, in the form of loops of various shapes and sizes, namely: between the cutaneous-fascia branches of the femoral and ilioinguinal nerves and the femoral and obturator nerves.
\end{abstract}

KEY WORDS: skin, innervation, femoral region, fetus, human

Wiad Lek. 2021;74(2):207-212

\section{INTRODUCTION}

Connective tissue formations, which are considered as a flexible soft skeleton of the human body, are important In the vital activity of the body. The soft skeleton contains formations with loose and dense connective tissue. Loose connective tissue is under the skin. More diverse formations of the soft skeleton are built of dense connective tissue - fascia, tendons, ligaments, capsule joints, fibrous sheaths of the neurovascular bundles and the like. The priority of fascia, in particular the fascia lata, is well-known in plastic surgery $[1,2,3]$. Elasticity, relative flexibility and fitness are inherent for fascia lata, which makes it possible to model it. However, data on the features of innervation of the fascia lata during the fetal period of human ontogenesis remain insufficiently studied in the research literature, and they remain fragmentary and contradictory [4].

Studies of individual authors were devoted to the variant anatomy of the lateral cutaneous nerve of the femur [510]. In particular, based on the lateral femoral cutaneous nerve topography study in 20 corpses by A. Hanna [9], it was found that coming out from the lateral edge of the psoas major muscle, the nerve crosses the iliac muscle and passes under the inguinal ligament at femur medial (6.5 $\mathrm{cm}$ ) or lateral $(6.0 \mathrm{~cm})$ of the anterior superior iliac spine. It is indicated that the lateral cutaneous nerve of the femur passes in the fascia canal. Also, in isolated cases, the lateral femoral cutaneous nerve runs along the anterior superior iliac spine, can be doubled, or start from the femoral nerve.

Usually, the lateral femoral cutaneous nerve runs laterally relative to the sartorius [6]. Some authors point to the bifurcation of the lateral femoral cutaneous nerve in anterior and posterior branches that innervate the skin of the anterolateral, lateral and posterior-lateral surfaces of the femur, including the greater trochanter region $[5,11,12]$.

Literature data suggest reports on the ultrasound anatomy of the cutaneous nerves of the extremities [12-14]. Thus, K.V. Chang et al. [13] describe the anterior femoral cutaneous nerve as a branch of the femoral nerve. The anterior femoral cutaneous nerve is divided into intermediate and anterior branches. The intermediate branch of the anterior femoral cutaneous nerve passes through the femur fascia lata, crosses the sartorius and innervates the skin of the anterior femoral and knee sections, and its anterior branch descends obliquely along the sartorius, penetrates the fascia in the distal third of the femur and provides innervation of the skin in the medial part of the anterior femoral region. Both branches of the anterior femoral cutaneous nerve can anastomose with lateral femoral cutaneous nerve, obturator nerve and patella branch of the saphenous nerve, forming the plexus [15].

The saphenous nerve is a direct extension of the femoral nerve and its terminal branch. It accompanies the femoral 
artery in the anterior femoral sulcus and in the adductor canal. The saphenous nerve exits the adductor canal along with the descending knee artery and penetrates the femoral fascia lata $[12,13]$.

Thus, the literature sources do not sufficiently cover the features of innervation of the skin of the anterior femoral region and the fascia lata during various periods of human ontogenesis, in particular in different age fetuses.

\section{THE AIM}

The aim is to find out the features of innervation of the skin of the anterior femoral region and the fascia lata during the fetal period of human development.

\section{MATERIALS AND METHODS}

The study was carried out on 64 preparations of the lower extremities of human fetuses of 4-10 months using macromicroscopic preparation and morphometry. Macromicroscopic preparation of the branches of the lumbar and sacral plexus was carried out using a binocular magnifier. First, the lumbar and sacral plexus nerves in the pelvic area were prepared, and then their cutaneous nerves or (and) cutaneous branches that go to the skin of the anterior and inner surfaces of the femoral region were prepared. It is necessary to note that in the pelvic region, the lumbar plexus nerves were prepared in two steps: first, the branches of the lumbar plexus were dissected with the preservation of the large lumbar muscle, and then after the latter was removed. Inguinal ligament was preserved in all cases. In order not to damage the cutaneous nerves of the femoral region of branches that intersect with each other, we followed the sequence of preparation of the nerves of the lumbar plexus. First, the iliohypogastric and ilio-inguinal nerves were prepared, then the branches of the ilio-inguinal and genitofemoral nerves were prepared, and after that, the lateral femoral cutaneous nerve, femoral and obturator nerves were prepared. The selected sequence of the cutaneous nerves of the anterior femoral region preparation made it possible to reveal a shift in the innervation zones, to establish overlap zones and other forms of variability by the topography of the nerve trunks and their branching in the skin of the femoral area. During the step-by-step preparation of branches of the lumbar plexus, variants of the structure and topography of cutaneous nerves or (and) cutaneous branches were found. Macropreparations of the skin nerves of the lower extremities of different age fetuses with anatomical variants were subject to photo documentation.

\section{RESULTS}

As a result of the study, the features of the fetal topography of individual cutaneous nerves of the anterior femoral region were identified and their connections were set, as well as the layered arrangement of their location. In our opinion, the layered arrangement of nerve trunks that provide innervation of the skin of the anterior femoral region is associated with the level of formation of the lumbar plexus nerves from the anterior branches of the spinal nerves, namely: the higher the level of the main nerve trunk origin, the more superficially its branches are placed in the subcutaneous fat and vice versa, the lower the nerve originates, the more deeply its branches are directed.

Taking into account the above, three cutaneous nerve sets can be distinguished in the anterior femoral region. One set is located on the anterior femoral surface, the anterior cutaneous branches of the femoral nerve, lateral femoral cutaneous nerve, the anterior cutaneous branch of the Iliohypogastric nerve, ilio-inguinal and genitofemoral nerves are involved in its formation (Fig. 1,2). Innervation of the skin of the upper third of the lateral femoral surface is provided by a complex of cutaneous nerves, which is mainly formed by the lateral cutaneous branch of the Iliohypogastric nerve and the lateral femoral cutaneous nerve. Also, the anterior cutaneous branches of the femoral nerve and the genitofemoral nerve participate in the innervation of the skin of the lateral femoral surface. It should be also noted that the lateral femoral cutaneous nerve below the inguinal ligament, as a rule, branches into 2-5 branches, which provide innervation of the lateral femoral surface skin to the knee (Fig. 3). At the same time, the cutaneous nerves or branches that innervate the skin of the anterolateral femoral surface, when exiting the femur, are mainly located behind the lateral and medial thirds of the inguinal ligament.

The following set of nerves is involved in the innervation of the medial femoral surface skin: 1-3 cutaneous branches of the obturator nerve, anterior cutaneous branches of the femoral nerve, saphenous and genitofemoral nerves (Fig. 4-6). Usually, 1-3 femoral branches depart from the latter to the skin of the upper medial surface of the femur under the inguinal ligament. The cutaneous branches of the obturator nerve mainly innervate the skin of the lower part of the femur inner surface. In the innervation of the femur skin of the thigh on the border of its front and rear surfaces can also participate lateral femoral cutaneous nerve, the femoral branch of the genitofemoral nerve, anterior cutaneous branches of the femoral nerve and cutaneous branch of the obturator nerve.

It was found that the lateral femoral cutaneous nerve formed connections with the anterior cutaneous branches of the femoral nerve at different levels of the femoral region. Anterior cutaneous branches of the femoral nerve, in addition to the above relations, also are connected with the cutaneous branches of the obturator nerve within the middle and lower thirds of the femoral internal surface, and the saphenous nerve in the femoral triangle region and anterior medial surface of the lower third of the femur in the region of the patella. The saphenous nerve is connected with the cutaneous branch of the obturator nerve at different levels: up to the adductor canal, in the canal itself, and in the lower third of the femur.

The superficial fascia on the anterior medial surface of the femur forms fascia cases for the cutaneous nerves and 


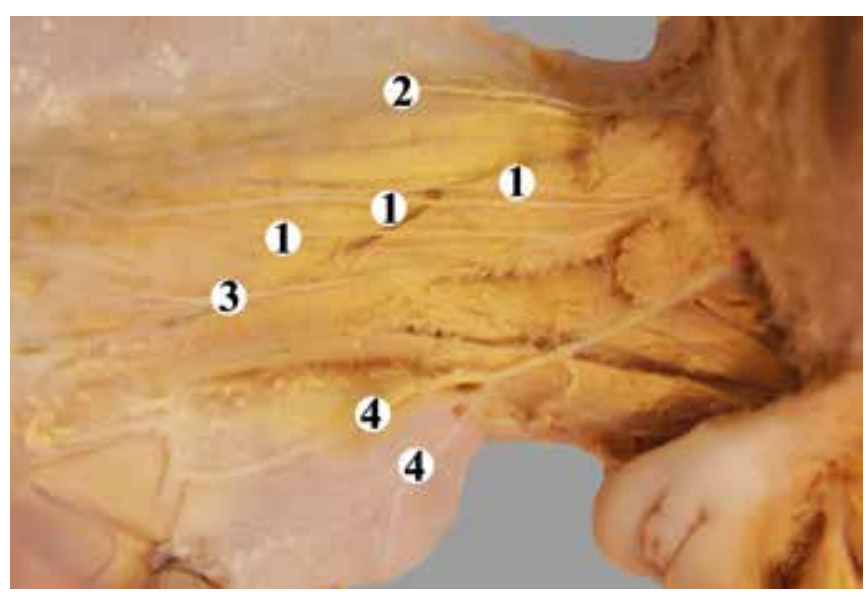

Fig. 1. Vessels and nerves of the right anterior femoral region of the female fetus $-285.0 \mathrm{~mm}$ of PCL. Macrospecimen. Mag. 2.4x:

1 - anterior cutaneous branches of the femoral nerve; 2 - lateral femoral cutaneous nerve; 3 - subcutaneous nerve; 4 - cutaneous branches of the femoral artery.

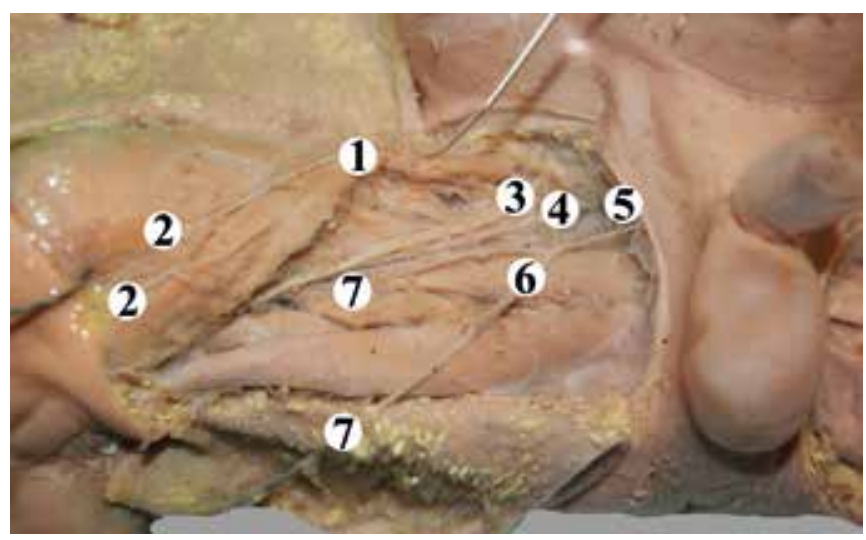

Fig. 3. Vessels and nerves of the right anterior femoral region of the male fetus $-265.0 \mathrm{~mm}$ of PCL. Macrospecimen. Mag. 2.5x:

1 - lateral femoral cutaneous nerve; 2 - branches of the lateral femoral cutaneous nerve; 3 - femoral nerve; 4 - femoral artery; 5 - femoral vein; 6 - great saphenous vein; 7 - cutaneous tributaries of the great saphenous vein.

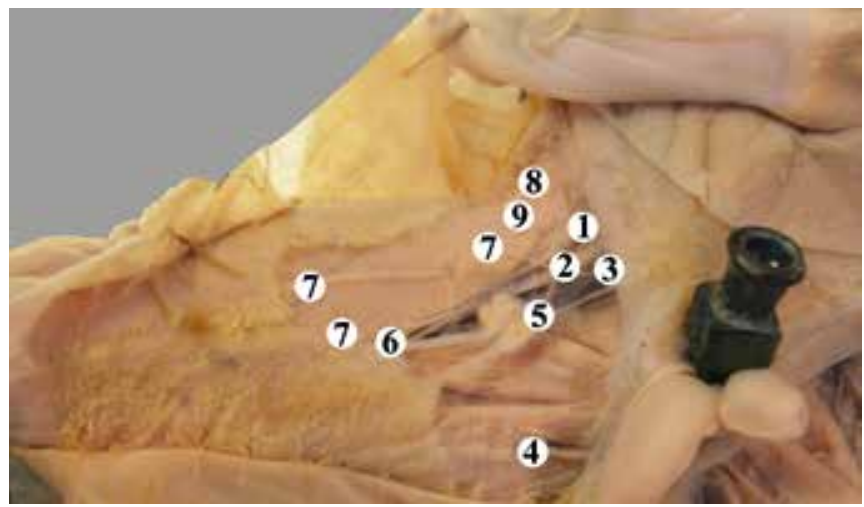

Fig. 5. Vessels and nerves of the right anterior femoral region of the male fetus $-240.0 \mathrm{~mm}$ of PCL. Macrospecimen. Mag. 2.1x:

1 -femoral nerve; 2 -femoral artery; 3 -femoral vein; 4 - obturator nerve; 5 - lymph node; 6 - great saphenous vein; 7 - branches of the femoral nerve; 8 - lateral femoral cutaneous nerve; 9 - connecting branch between the lateral femoral cutaneous nerve and the femoral nerve.

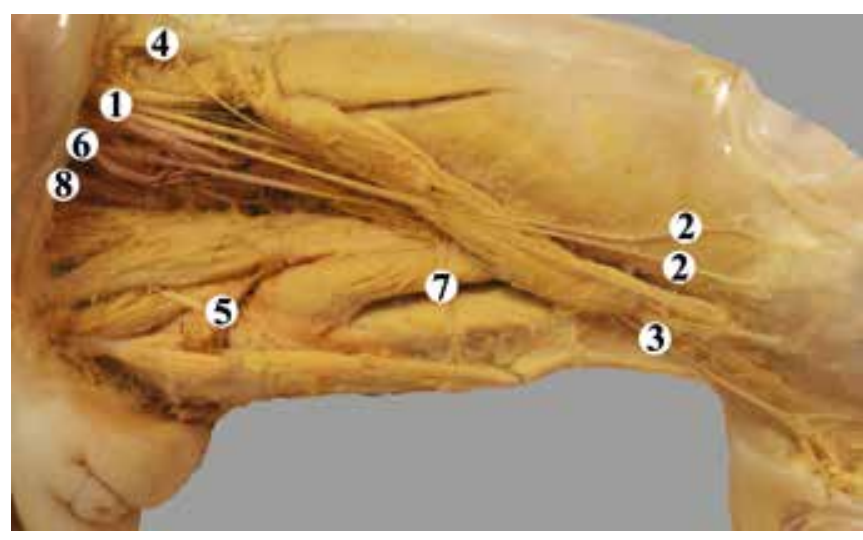

Fig. 2. Vessels and nerves of the left anterior femoral region of the female fetus $-285.0 \mathrm{~mm}$ of PCL. Macrospecimen. Mag. 2.1x:

1 - femoral nerve; 2 - anterior cutaneous branches of the femoral nerve; 3-subcutaneous nerve; 4 - lateral femoral cutaneous nerve; 5 - obturator nerve; 6 - femoral artery; 7 - muscular branch of the femoral artery; 8 - femoral vein.

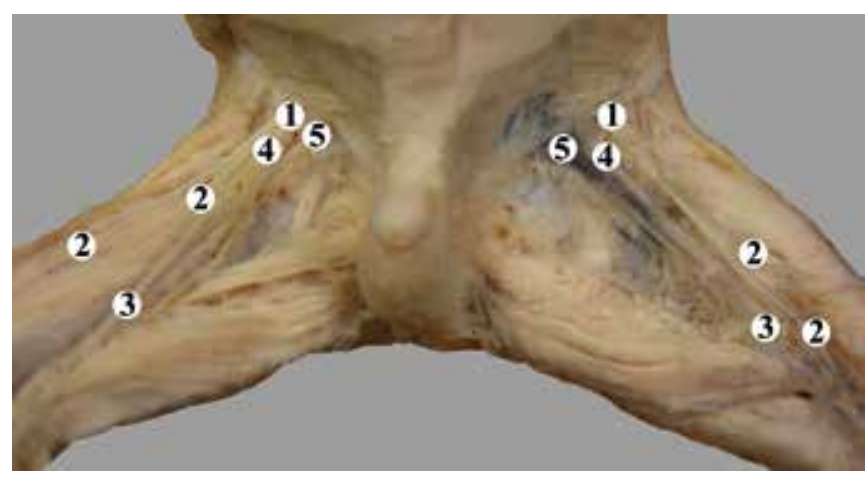

Fig. 4. Vessels and nerves of the anterior femoral regions of the male fetus $272.0 \mathrm{~mm}$ PCL. Macrospecimen. Mag. $1.8 \mathrm{x}$ :

1 - femoral nerve; 2 - anterior cutaneous branches of the femoral nerve; 3 - subcutaneous nerve; 4 - femoral artery; 5 - femoral vein.

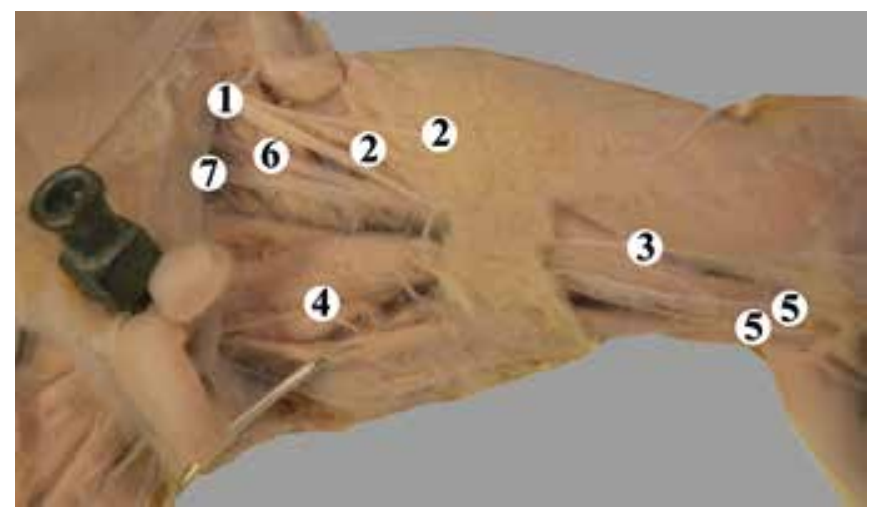

Fig. 6. Vessels and nerves of the left anterior femoral region of the male fetus $-240.0 \mathrm{~mm}$ of PCL. Macrospecimen. Mag. 2.2x:

1 - femoral nerve; 2 - anterior cutaneous branches of the femoral nerve; 3- subcutaneous nerve; 4 - obturator nerve; 5 - obturator nerve branches; 6 -femoral artery; 7 -femoral vein.

the great saphenous vein. Within the upper third of the femoral triangle, the superficial fascia loosely fuses with the inguinal ligament and the surface plate of the femur fascia lata. Fascia sheath of the great saphenous vein in 


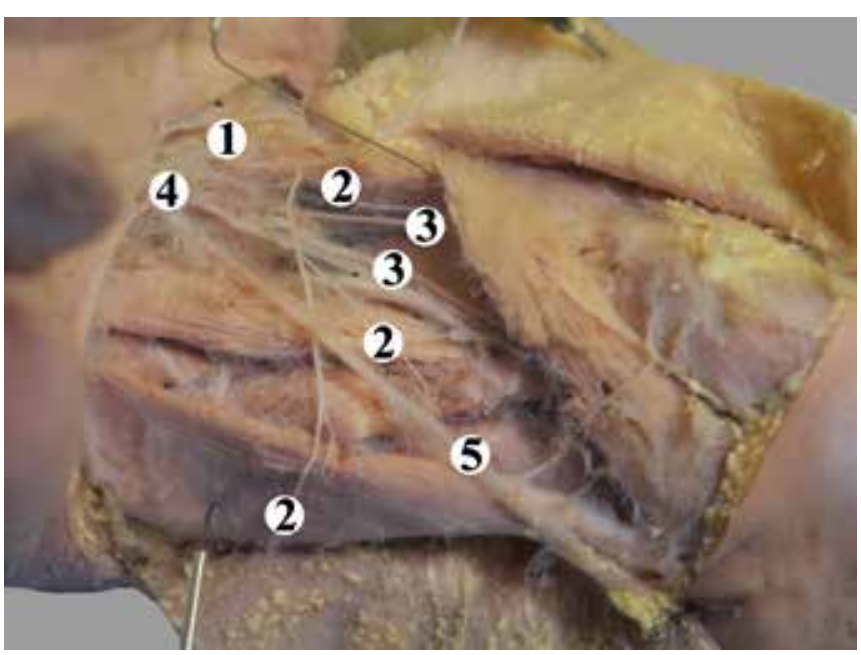

Fig. 7. Vessels and nerves of the left anterior femoral region of the male fetus $-265.0 \mathrm{~mm}$ of PCL. Macrospecimen. Mag. $2.3 \mathrm{x}$ :

1 -ilio-inguinal nerve; 2 - cutaneous branches of the ilio-inguinal nerve; 3 - branches of the femoral nerve; 4 - femoral vein; 5 - great saphenous vein.

the upper third of the femur formed by splitting of the superficial fascia or the fascia lata plate, and the medial and lower thirds of the femur that vein is located in the splitting of the plates of the fascia lata. Superficial inguinal lymph nodes are located on both sides of the great saphenous vein at its entry to the femoral vein. The fascia cases of the latter are loosely connected to the fascial sheath of the great saphenous vein. It should be noted that over the fetal period of ontogenesis, the fascia lata along its length is of unequal structure. In the proximal part of the anterior femoral region, the fascia lata is poorly developed in different age fetuses. In the area of the femoral triangle, when moving from the sartorius to the adductor muscles, the fascia lata is divided into superficial and deep plates and forms fascial cases for the superficially located femoral muscles: muscle tensioner of the fascia lata, sartorius, thin muscle and rectus femoris. The surface plate of the fascia lata, in turn, is divided into several thin, loose plates that can not be distinguished, because fat inclusions are found in the surface fascia and the surface plate of the fascia lata. There is an oval fossa in the surface plate of the fascia lata. In the studied fetuses, the fascia lata plates are transparent and loose, with the exception of the outer surface of the femur, where the fascia lata is slightly compacted and forms an iliotibial strand. In 8-10 month fetuses, the broad fascia throughout the posterior femoral region, primarily within the popliteal fossa, is strengthened by bundles of fibers, mainly in a transverse direction.

The branches of the lumbar and sacral plexus are the source of the fascia lata innervation. In particular, 3-6 cutaneous fascial branches extend from the femoral nerve below the inguinal ligament, which penetrate the fascia lata and branch out within the anteromedial femoral surface. 2-3 branches are directed to the fascia and skin of the upper third of the medial femur surface from the ilio-inguinal nerve below the inguinal ligament (Fig. 7).

As a rule, 1-2 branches and sometimes three branches of the obturator nerve are involved in the innervation of the skin of the medial femoral surface. In some cases, 1-2 skin-fascial branches of the obturator nerve reach the level of the lower third of the medial femur surface.

The lateral femoral cutaneous nerve below the anterior superior iliac spine gives off 2-3 branches involved in the innervation of the fascia lata and skin of the lateral and partially anterior femoral surfaces. 2-4 branches of the posterior cutaneous femoral nerve are involved in the innervation of the fascia lata and skin of the posterior femoral region.

The terminal branches of the femoral nerve overlap the cutaneous-fascial branch of the obturator nerve, the branches of the lateral femoral cutaneous nerve, as well as the branches of the iliac-inguinal nerve on the lateral surface - the branches of the lateral femoral cutaneous nerve, on the medial femoral branch surface. The latter also forms "overlapping zones" with branches of the posterior cutaneous nerve on the posterior femoral surface, and in some cases, in the area of the lateral femoral surface. Together with the venous and arterial vessels that accompany the branching of the above-mentioned nerves, a neurovascular network is formed in the thickness of the connective tissue of the fascia lata.

Anastomoses in the form of loops of various shapes and sizes were found in the anterior-medial femoral surface region of the studied fetuses, namely: between the cutaneous-fascia branches of the femoral and ilioinguinal nerves (within the upper third) and the femoral and obturator nerves (within the middle third).

\section{DISCUSSION}

It should be noted that the textbooks and manuals on anatomy indicate that in the skin of the lateral and anterior-lateral femoral surfaces the lateral femoral cutaneous nerve divides, the skin of the upper-medial femoral surface under the inguinal ligament is innervated by the femoral branch of the genitofemoral nerve, the skin of the lower portion of the medial femoral surface - by the cutaneous branch of the obturator nerve and anterior cutaneous branches of the femoral nerve innervate the anterior femoral surface skin $[9,16]$. However, as a result of the study, anatomical variability in the innervation of the skin of the anterior femoral region was found. It was also found that in human fetuses, not only the lateral cutaneous femoral nerve but in most cases the branches of other nerves of the lumbar plexus, except for the obturator nerve, are directed to the skin of the anterior-lateral femur surface. The innervation of the skin of the anterior-lateral femur surface is provided by a cutaneous nerve complex: the femoral nerve, the lateral femoral cutaneous nerve, iliohypogastric, ilioinguinal and genitofemoral nerves. The innervation of the medial femur surface is provided by the following nerve complex: obturator, femoral, saphenous and genitofemoral nerves. Based on a thorough anatomical study of the branching zones of the cutaneous nerves in the anterior femoral region and the fascia lata, 
both the displacement of the innervation zones and the overlap zones were set. It was found that as a result of branching of the ilioinguinal branches of the femoral, obturator, iliac-inguinal, lateral and posterior cutaneous nerves of the thigh, "overlapping zones" of one nerve by the other are formed on the entire surface of the fascia lata. Therefore, the terminal branches of these nerves involved in the innervation of the fascia lata do not have a well-defined topography.

The obtained data on the variant anatomy of the cutaneous nerves and the cutaneous-fascial branches of the nerves of the anterior femoral region should be taken into account by surgeons during surgical interventions. As K.A. Tomaszewski et al. [7] stress that knowing the possible options for the topography of the lateral cutaneous nerve will help reduce the risk of nerve damage during surgery within the inguinal and femoral region, in particular during hip replacement and inguinal herniotomies.

The results of the study are also of great practical importance for neurosurgery, neurology and reflexology.

\section{CONCLUSIONS}

In human fetuses, anatomical variability of the cutaneous nerves and cutaneous branches of the anterior femoral nerve region was established, and three sets of cutaneous nerves were identified.

Anastomoses were found between the cutaneous nerves, in the form of loops of various shapes and sizes, namely: between the cutaneous-fascia branches of the femoral and ilioinguinal nerves (within the upper third) and the femoral and obturator nerves (within the middle third).

Taking into account the fact that the terminal branches of adjacent cutaneous nerves of the femoral region intersect and overlap, innervation bypasses are formed, due to which, in case of possible damage to one of the nerves, its insufficiency is compensated to a certain extent.

In human fetuses, innervation of the fascia lata in the anterio-medial femoral surface is provided by branches of the femoral, obturator, and ilio-inguinal nerves, and in the posterior-lateral surface of the femur - branches of the posterior and lateral cutaneous nerves of the femur.

Connections and complexes of femoral cutaneous nerves, as well as overlapping and displacement zones are compensatory mechanisms in the peripheral nervous system and are observed not only between ontogenically related nerves, but also nerves of various segmental affiliation.

\section{REFERENCES}

1. Hosny H., Abdelsattar A.H. Fascial Graft Repair of Wide Bilateral Cleft Lip Deformity. J Craniofac Surg. 2018; 29(1): e1-e4. doi: 10.1097/SCS. 0000000000003960.

2. Szotek S., Dawidowicz J., Eyden B. et al. Morphological features of fascia lata in relation to fascia diseases. Ultrastruct Pathol. 2016; 40(6): 297-310.

3. Peláez Mata D., Alvarez Zapico J.A., Gutiérrez Segura C. et al. Fascia lata transplant from cadaveric donor in the reconstruction of abdominal wall defects in children. Cir Pediatr. 2001; 14(1): 28-30.
4. Beger 0., Koç T., Beger B. et al. Morphometric properties of the tensor fascia lata muscle in human foetuses. Folia Morphol (Warsz). 2018; 77(3): 498-502. doi: 10.5603/FM.a2018.0007.

5. Grothaus M.C., Holt M., Mekhail A.O. et al. Lateral femoral cutaneous nerve: an anatomic study. Clin Orthop Relat Res. 2005; 437: 164-168.

6. Mischkowski R.A., Selbach I., Neugebauer J. et al. Lateral femoral cutaneous nerve and iliac crest bone grafts-anatomical and clinical considerations. Int J Oral Maxillofac Surg. 2006; 35(4): 366-72.

7. Tomaszewski K.A., Popieluszko P., Henry B.M. et al. The surgical anatomy of the lateral femoral cutaneous nerve in the inguinal region: a metaanalysis. Hernia. 2016;20(5):649-57. doi: 10.1007/ s10029-016-1493-7.

8. Reinpold W., Schroeder A.D., Schroeder M. et al. Retroperitoneal anatomy of the iliohypogastric, ilioinguinal, genitofemoral, and lateral femoral cutaneous nerve: consequences for prevention and treatment of chronic inguinodynia. Hernia. 2015; 19(4): 539-48. doi: 10.1007/ s10029-015-1396-z.

9. Hanna A. The lateral femoral cutaneous nerve canal. J Neurosurg. 2017; 126(3): 972-978. doi: 10.3171/2016.1.JNS152262.

10. LeeS.H.,Shin K.J., GilY.C. et al. Anatomy of the lateral femoral cutaneous nerve relevant to clinical findings in meralgia paresthetica. Muscle Nerve. 2017; 55(5): 646-650. doi: 10.1002/mus.25382.

11. Anloague P.A., Huijbregts P. Anatomical variations of the lumbar plexus: a descriptive anatomy study with proposed clinical implications. J Man Manip Ther. 2009; 17: 107-114.

12. Hung C.Y., Hsiao M.Y., Ozcakar L. et al. Sonographic Tracking of the Lower Limb Peripheral Nerves: A Pictorial Essay and Video Demonstration. Am. J. Phys. Med. Rehabil. 2016; 95: 698-708.

13. Chang K.V., Mezian K., Naňka 0. et al. Ultrasound Imaging for the Cutaneous Nerves of the Extremities and Relevant Entrapment Syndromes: From Anatomy to Clinical Implications. J Clin Med. 2018; 7(11): 457. doi: 10.3390/jcm7110457

14. Kowalska B., Sudoł-Szopińska I. Ultrasound assessment of selected peripheral nerves pathologies. Part II: Entrapment neuropathies of the lower limb. J Ultrason. 2012; 12(51): 463-471. doi: 10.15557/ JoU.2012.0033

15. Pivec C., Bodner G., Mayer J.A. et al. Novel Demonstration of the Anterior Femoral Cutaneous Nerves using Ultrasound. Ultraschall in Med. 2018; 39:318-327.

16. Petchprapa C.N., Rosenberg Z.S., Sconfienza L.M. et al. MR imaging of entrapment neuropathies of the lower extremity. Part 1. The pelvis and hip. Radiographics. 2010; 30(4): 983-1000. doi: 10.1148/rg.304095135.

The study is a part of the planned complex topic at the Department of Histology, Cytology and Embryology of the HSEI of Ukraine Bukovynian State Medical University named "Regularities of Morphogenesis, Structural and Functional Features of Tissues and Organs in Human Ontogenesis", state registration No. 0116 U002938.

\section{ORCID and contributionship:}

Pavlina V. Hryhorieva: 0000-0003-2400-0569 ${ }^{A, D}$

Tatiana V. Khmara: 0000-0001-8023-5181 ${ }^{F}$

Alina O. Palamar: 0000-0002-8935-3552 ${ }^{B}$

Tetyana B. Sykyrytska: 0000-0002-9386-8524 ${ }^{C}$

Maryna Yu. Leka: 0000-0003-3397-4605 ${ }^{\mathrm{E}}$

\section{Conflict of interest:}

The Authors declare no conflict of interest. 


\section{CORRESPONDING AUTHOR}

\section{Tatiana V. Khmara}

Higher State Educational Establishment of

Ukraine "Bukovinian State Medical University"

2 Teatralna sq., 58001 Chernivtsi, Ukraine

tel: +380997516550

e-mail: khmara.tv.6@gmail.com

Received: 04.03 .2020

Accepted: 18.11 .2020

A - Work concept and design, B - Data collection and analysis, C - Responsibility for statistical analysis,

$\mathbf{D}$-Writing the article, $\mathbf{E}$-Critical review, $\mathbf{F}$ - Final approval of the article 